\title{
Ethanol Induces Long-Term Facilitation of NR2B-NMDA Receptor Activity in the Dorsal Striatum: Implications for Alcohol Drinking Behavior
}

\author{
Jun Wang, ${ }^{1}$ Sebastien Carnicella, ${ }^{1}$ Khanhky Phamluong, ${ }^{1}$ Jerome Jeanblanc, ${ }^{1}$ Jennifer A. Ronesi, ${ }^{3}$ Nadia Chaudhri, ${ }^{1}$ \\ Patricia H. Janak, ${ }^{1,2}$ David M. Lovinger, ${ }^{3}$ and Dorit Ron ${ }^{1,2}$ \\ ${ }^{1}$ Ernest Gallo Research Center, ${ }^{2}$ Department of Neurology, University of California, San Francisco, Emeryville, California 94608, and ${ }^{3}$ Laboratory for \\ Integrative Neuroscience, National Institute on Alcohol Abuse and Alcoholism-National Institutes of Health, Bethesda, Maryland 20892
}

\begin{abstract}
Addiction is characterized by compulsive alcohol or drug taking and seeking, and the dorsal striatum has been implicated in such maladaptive persistent habits. The NMDA receptor (NMDAR), which is a major target of alcohol, is implicated in striatal-based habit learning. We found that, in the dorsal striatum, alcohol (ethanol) exposure produced an increase in the phosphorylation of the NR2B subunit of the NMDAR, and a corresponding increase in the activity of Fyn kinase, which phosphorylates NR2B. We further observed an ethanol-mediated long-term facilitation (LTF) of the activity of NR2B-containing NMDARs (NR2B-NMDARs) in the dorsal striatum. This LTF is Fyn kinase dependent, because it was observed in Fyn wild-type but not in Fyn knock-out mice. Importantly, none of these biochemical and physiological changes was observed in the ventral striatum. Finally, dorsal but not ventral striatum infusion of a Fyn or NR2B-NMDAR inhibitor reduced rat operant self-administration of ethanol. Our results suggest that the Fyn-mediated phosphorylation and LTF of NR2B-NMDAR activity in the dorsal striatum after exposure to ethanol may underlie aberrant plasticity that contributes to mechanisms underlying alcohol drinking behavior.
\end{abstract}

Key words: accumbens; addiction; drinking; Fyn; NMDA receptors; self-administration; striatum; synaptic plasticity

\section{Introduction}

The dorsal striatum, a brain region that controls movement (Graybiel et al., 1994), has been implicated in mediating the formation of goal-directed responses and behavioral habits (Yin and Knowlton, 2006). Animal and human studies in recent years suggest that addiction is a form of persistent habit that is controlled by the dorsal striatum (Berke and Hyman, 2000; Everitt and Robbins, 2005). For example, cocaine-paired conditioned stimuli increase dopamine release in the dorsal striatum of rats trained extensively under a second-order schedule (Ito et al., 2002). Infusion of a dopamine receptor antagonist into the dorsal striatum reduces cocaine seeking of rats under such a second-order schedule (Vanderschuren et al., 2005). Finally, recent human imaging studies implicate increases in dopamine in the dorsal striatum in craving for cocaine (Volkow et al., 2006). Together, these data suggest that the dorsal striatum plays a crucial role in habitual learning and memory processes that may underlie addictive phenotypes such as compulsive drug and alcohol administration.

Received 0ct. 31, 2006; revised March 2, 2007; accepted March 5, 2007.

This work was supported by National Institute on Alcohol Abuse and Alcoholism (R01AA/MH13438-01A1) (D.R.), by the Division of Intramural Clinical and Basic Research (J.A.R., D.M.L.), and by funds provided by the state of California for medical research on alcohol and substance abuse through the University of California, San Francisco (D.R., P.H.J.).

Correspondence should be addressed to Dr. Dorit Ron, Ernest Gallo Research Center, 5858 Horton Street, Suite 200, Emeryville, CA 94608. E-mail: dorit.ron@ucsf.edu.

D0I:10.1523/JNEUROSCI.4749-06.2007

Copyright $\odot 2007$ Society for Neuroscience $\quad$ 0270-6474/07/273593-10\$15.00/0
The dorsal striatum has been suggested as a site of aberrant synaptic plasticity induced by exposure to drugs of abuse and ethanol (Gerdeman et al., 2003). The NMDA receptor (NMDAR) has long been known to be crucial for the induction of synaptic plasticity (Malenka and Nicoll, 1999; Martin et al., 2000), and the NMDAR is required for the induction of long-term potentiation (LTP) in the dorsal striatum (Calabresi et al., 1992; Partridge et al., 2000; Kerr and Wickens, 2001; Gerdeman et al., 2003). The NMDAR is also important for learning and memory in the dorsal striatum. For example, intradorsal striatal injection of an NMDAR antagonist impairs memory of rats in a stimulus-response task (Packard and Teather, 1997), and peripheral posttraining injection of a NMDAR antagonist also impairs such memories (Teather et al., 2001).

The NMDAR is a major target of ethanol (Kumari and Ticku, 2000; Ron, 2004), and this receptor has been implicated in ethanol-associated phenotypes such as tolerance, dependence, withdrawal, craving, and relapse (Krystal et al., 2003). For example, NMDAR antagonists block the development of rapid tolerance to ethanol (Khanna et al., 1993), decrease ethanol intake (Holter et al., 2000), and reduce the intensity of ethanol withdrawal symptoms (Bisaga et al., 2000). In addition, administration of a NMDAR antagonist decreases ethanol intake in a rodent relapse model (Holter et al., 2000). However, the brain regions that produce these phenotypes are not well understood. The NMDAR consists of an obligatory NR1 subunit and NR2(A-D) subunits (Sucher et al., 1996). The major NR2 subunits are NR2A 
and NR2B, and these subunits are expressed in the striatum (Kawakami et al., 2003). NR2A- and NR2B-containing NMDARs possess different pharmacological properties (Cull-Candy and Leszkiewicz, 2004). The NR2 subunits are phosphorylated by the Src family protein tyrosine kinases (PTKs) Fyn and Src (Salter and Kalia, 2004), leading to upregulation of channel function (Salter and Kalia, 2004). The actions of ethanol on the NMDAR are also regulated by phosphorylation of the subunits (Ron, 2004).

Because the NMDAR plays a role in dorsal striatal learning and memory, and because the NMDAR is an important mediator of the actions of ethanol, we chose to determine whether, and how, ethanol exposure modulates NMDAR phosphorylation state and function in the dorsal striatum. We observed long-term facilitation (LTF) of NMDAR transmission in response to ethanol application in the dorsal striatum that was mediated by Fyn phosphorylation of the NR2B subunit of the receptor. Importantly, we provide data to suggest that this pathway contributes to dorsal striatal-mediated self-administration of ethanol.

\section{Materials and Methods \\ Reagents}

The polyclonal anti-NR2A and anti-NR2B antibodies and the monoclonal anti-Fyn and anti-RACK1 antibodies were purchased from Santa Cruz Biotechnologies (Santa Cruz, CA). The monoclonal antiphosphotyrosine antibodies were purchased from Transduction Laboratories (Lexington, KY). The monoclonal anti-Src antibodies were purchased from Upstate Biotechnologies (Lake Placid, NY). Polyclonal anti-[pY417/418] Fyn/Src antibodies were purchased from BioSource International (Camarillo, CA). The polyclonal anti-[pY1252]NR2B antibodies were generated as described previously (Takasu et al., 2002). 4-Amino-5-(4-chlorophenyl)-7-( $t$-butyl)pyrazolo[3,4-D]pyrimidine (PP2) and 4-amino-7-phenylpyrazol[3,4-D]pyrimidine (PP3) were purchased from Calbiochem (La Jolla, CA). Protease inhibitor mixture was purchased from Roche (Basel, Switzerland). The phosphatase inhibitor mixture, picrotoxin, and sodium orthovanadate were purchased from Sigma (St. Louis, MO). 2,3-Dioxo-6-nitro-1,2,3,4-tetrahydrobenzo [f] quinoxaline-7-sulfonamide disodium salt (NBQX) and the NR2BNMDAR inhibitors $(\alpha R, \beta S)-\alpha$-(4-hydroxyphenyl)- $\beta$-methyl-4(phenylmethyl)-1-piperidinepropanol hydrochloride (Ro 25-6981) and ifenprodil were obtained from Tocris (Ellisville, MO).

\section{Animals}

Male Sprague Dawley rats (3-4 weeks of age) and male Long-Evans rats (350-450 $\mathrm{g}$ at the time of surgery) were obtained from Harlan (Indianapolis, IN). Male C57BL/6 mice (5-7 weeks of age) were purchased from The Jackson Laboratory (Bar Harbor, ME). Generation of Fyn knock-out mice and their littermates has been described previously (Yaka et al., 2003c). Briefly, mating pairs of Fyn ${ }^{-1-}$ (C57BL/6;129) male and C57BL/6 female mice purchased from The Jackson Laboratory were used to generate Fyn ${ }^{-1-}$ and Fyn ${ }^{+/+}$mice. Mouse genotypes were determined by PCR analysis of products derived from tail DNA and were used for experiments at 3-5 weeks of age. Animals used in these studies were housed under a $12 \mathrm{~h}$ light/dark cycle, with lights on at 7:00 A.M. and food and water available ad libitum. The electrophysiological and biochemical experiments were done in Sprague Dawley rats, whereas LongEvens rats were used for the behavioral experiments. Sprague Dawley rats are the most commonly used rats in electrophysiological and biochemical studies. Sprague Dawley rats are docile and have excellent reproductive performance and maternal characteristics. However, Long-Evans rats are more suitable for behavioral studies. Compared with pigmented Long-Evans rats, albino Sprague Dawley rats have poorer visual acuity (Prusky et al., 2002), have poorer learning (Fregly and Rowland, 1992; Harker and Whishaw, 2002), and have motor impairments (Whishaw et al., 2003). Finally, Sprague Dawley rats do not drink appreciable amounts of unadulterated ethanol. In contrast, Long-Evans rats show a good range of ethanol intake (Khanna et al., 1990). All animal procedures in this report were approved by the Gallo Center Institutional Animal Care and Use Committee and were conducted in agreement with the Guide for the Care and Use of Laboratory Animals, National Research Council, 1996.

Preparation of slices and homogenates for biochemical analyses Coronal slices $(250 \mu \mathrm{m})$ were dissected and maintained for at least $2 \mathrm{~h}$ in artificial CSF (aCSF) that contained the following (in mM): $126 \mathrm{NaCl}, 1.2$ $\mathrm{KCl}, 1.2 \mathrm{NaH}_{2} \mathrm{PO}_{4}, 0.01 \mathrm{MgCl}_{2}, 2.4 \mathrm{CaCl}_{2}, 18 \mathrm{NaHCO}_{3}$, and 11 glucose, and saturated with $95 \% \mathrm{O}_{2} / 5 \% \mathrm{CO}_{2}$ at $25^{\circ} \mathrm{C}$. After recovery, slices were treated and homogenized in homogenization buffer that contained the following (in mM): 320 sucrose, 10 Tris- $\mathrm{HCl}, 10$ EDTA, 10 EGTA, protease inhibitor mixture, and phosphatase inhibitor mixture, adjusted to $\mathrm{pH}$ 7.4. Homogenates were centrifuged at $5000 \times g$ for $3 \mathrm{~min}$. The pellet was resuspended in solubilization buffer containing the following (in $\mathrm{mm}$ ): 10 Tris-HCl, 10 EDTA, 10 EGTA, protease inhibitor mixture, and phosphatase inhibitor mixture, adjusted to $\mathrm{pH} 7.4$, and including $1 \%$ Triton X-100 for phosphorylation assays or $1 \%$ deoxycholate for association assays.

\section{Immunoprecipitation}

Slice homogenates were precleared by incubation with protein G-agarose (Invitrogen, Carlsbad, CA) for $2 \mathrm{~h}$ at $4^{\circ} \mathrm{C}$. The samples were centrifuged and protein quantity was determined using BCA (bicinchoninic acid) (Pierce, Rockford, IL). Immunoprecipitation was performed by combining $5 \mu \mathrm{g}$ of the appropriate antibody with $\sim 0.5 \mathrm{mg}$ of protein diluted in $1 \times$ immunoprecipitation buffer, containing the following (in $\mathrm{mM}$ ): 150 $\mathrm{NaCl}, 10$ Tris-HCl, 1 EDTA, 1 EGTA, 1\% Triton X-100, protease and phosphatase inhibitor mixtures, adjusted to $\mathrm{pH}$ 7.4. After overnight incubation at $4^{\circ} \mathrm{C}$, protein $\mathrm{G}$-agarose was added, and the mixture was incubated at $4^{\circ} \mathrm{C}$ for $2 \mathrm{~h}$. The protein $\mathrm{G}$ was washed extensively, and pellets were resolved on a 10\% SDS-PAGE gel.

\section{Western blot analysis}

Samples were separated by 4-12\% SDS-PAGE (Bis-Tris Gel system; Invitrogen) at $160 \mathrm{~V}$ for $60 \mathrm{~min}$. Proteins were then transferred onto a nitrocellulose membrane (Millipore, Billerica, MA). Membranes were blocked in milk solution (5\% milk, PBS, $0.05 \%$ Tween 20 ) and incubated with specific primary antibodies, followed by horseradish peroxidaseconjugated secondary antibodies. Immunoreactivity was detected by enhanced chemiluminescent reaction and processed using the Typhoon PhosphorImager (Amersham Biosciences, Piscataway, NJ). Results were quantified with ImageQuant 5.0 (Amersham Biosciences).

\section{Electrophysiology}

Striatal slices were prepared from rats and transgenic mice using techniques similar to those described previously (Ronesi et al., 2004). Briefly, animals were anesthetized with halothane and decapitated as per University of California, San Francisco, animal care guidelines. Coronal sections of the striatum $(250-300 \mu \mathrm{m})$ were cut with a vibratome (Leica, Nussloch, Germany) in an ice-cold cutting solution containing the following (in $\mathrm{mm}$ ): $40 \mathrm{NaCl}, 85$ choline chloride, $4 \mathrm{KCl}, 1.25 \mathrm{NaH}_{2} \mathrm{PO}_{4}, 26$ $\mathrm{NaHCO}_{3}, 0.5 \mathrm{CaCl}_{2}, 7 \mathrm{MgCl}_{2}, 10$ glucose, 1 sodium ascorbate, and 3 sodium pyruvate, saturated with $95 \% \mathrm{O}_{2}-5 \% \mathrm{CO}_{2}$. Slices were then incubated in the same solution at $32^{\circ} \mathrm{C}$ for $45 \mathrm{~min}$ before transferring to a chamber at room temperature. This chamber contained a normal external solution composed of the following (in $\mathrm{mM}$ ): $125 \mathrm{NaCl}, 2.5 \mathrm{KCl}, 2.5$ $\mathrm{CaCl}_{2}, 1.3 \mathrm{MgCl}_{2}, 1.25 \mathrm{NaH}_{2} \mathrm{PO}_{4}, 25 \mathrm{NaHCO}_{3}$, and 10 glucose, saturated with $95 \% \mathrm{O}_{2}-5 \% \mathrm{CO}_{2}$. For recordings of NMDAR-mediated synaptic transmission, picrotoxin $(100 \mu \mathrm{M}), \mathrm{NBQX}(10 \mu \mathrm{M})$, and a low concentration of $\mathrm{Mg}^{2+}(0.05 \mathrm{mM})$ were used in the external solution to block $\mathrm{GABA}_{\mathrm{A}}$ receptor $\left(\mathrm{GABA}_{\mathrm{A}} \mathrm{R}\right)$-mediated inhibitory synaptic transmission and AMPA/kainate receptor (AMPAR/KAR)-mediated excitatory synaptic transmission, and to release the $\mathrm{Mg}^{2+}$-dependent blockade of NMDAR activity, respectively. For recording of AMPAR-mediated synaptic transmission, picrotoxin $(100 \mu \mathrm{M})$ and a normal concentration of $\mathrm{Mg}^{2+}(1.3 \mathrm{~mm})$ were used in the external solution to block $\mathrm{GABA}_{\mathrm{A}} \mathrm{R}$ - and NMDAR-mediated synaptic transmission, respectively. To isolate the process that is mediated solely via tyrosine phosphorylation, the experiments were conducted in the presence of the tyrosine phosphatase inhib- 
itor sodium orthovanadate $(200 \mu \mathrm{M})$. However, significant long-term facilitation of NMDAR-mediated synaptic transmission was also observed in the absence of the phosphatase inhibitor (supplemental Fig. 1, available at www.jneurosci.org as supplemental material). Slices were superfused at 3-4 $\mathrm{ml} / \mathrm{min}$, and cells were visualized using infrared differential interference contrast video microscopy (BX50WI; Olympus, Tokyo, Japan). Whole-cell voltage-clamp recordings were made using a MultiClamp 700A amplifier (Molecular Devices, Union City, CA). Electrodes (4-6 M $\Omega$ ) contained the following (in $\mathrm{mM}$ ): 115 cesium methanesulfonate, 15 HEPES, 0.6 EGTA, 8 TEA-Cl, 4 MgATP, 0.3 NaGTP, and $7 \mathrm{Na}_{2} \mathrm{CrPO}_{4}$, pH 7.2-7.3, with an osmolarity of 270-280 mOsm. Series resistance and input resistance were monitored on-line. Stimulation of inputs to the striatum was achieved with tungsten bipolar electrodes (FHC, Bowdoinham, ME) that were placed in the dorsal or ventral striatum (the medial shell of the nucleus accumbens) and were used to stimulate excitatory afferents at $0.05 \mathrm{~Hz}$. Neurons were voltage clamped at $-70 \mathrm{mV}$. EPSCs were filtered at $2 \mathrm{kHz}$, digitized at $5-10 \mathrm{kHz}$, and collected on-line using pClamp 9 (Molecular Devices). After blockade of $\mathrm{GABA}_{\mathrm{A}} \mathrm{R}$ by picrotoxin and of AMPA/KAR by NBQX, slow decaying EPSCs were recorded in a low $\mathrm{Mg}^{2+}$ solution (see Fig. $2 A$, inset). These EPSCs were abolished by the NMDAR antagonist APV ( $50 \mu \mathrm{M})$ (data not shown), confirming that they were mediated by NMDARs.

\section{Operant ethanol self-administration}

The self-administration paradigm is as described previously (Jeanblanc et al., 2006). Briefly, the rats were habituated to drink ethanol in their home cage using different solutions of $10 \%$ ethanol mixed with decreasing concentrations of sucrose $(10,5$, and $0 \%)$. After 3 weeks, the rats started operant ethanol self-administration training. The selfadministration chambers contain two levers: an active lever, for which presses result in delivery of $0.1 \mathrm{ml}$ of fluid reward (a 10\% ethanol solution), and an inactive lever, for which responses are counted but no programmed events occur. After $4 \mathrm{~d}$ under a fixed ratio 1 (FR1) (one press delivers one reward), the rats were trained on a FR3 schedule (three presses are needed to receive one reward), $5 \mathrm{~d}$ a week in $60 \mathrm{~min}$ sessions. After 2 months, surgery to implant the cannulas was conducted.

\section{Sucrose self-administration}

Rats were initially trained under FR1 using $8 \%$ sucrose during two overnight sessions. Next, the FR schedule was progressively increased to FR3 and sucrose concentration was progressively decreased to $2 \%, 5 \mathrm{~d}$ a week in 60 min sessions.

\section{Surgery and microinjection of PP2, PP3, and ifenprodil}

Male Long-Evans rats (350-450 $\mathrm{g}$ at the time of the surgery) were anesthetized continuously with isoflurane (Baxter Health Care Corporation, Deerfield, IL). Bilateral guide cannulas (C235G-2.0, 26 gauge; Plastics One, Roanoke, VA) were implanted into the dorsal striatum $(1.0 \mathrm{~mm}$ anterior to bregma, $3.2 \mathrm{~mm}$ mediolateral, $3.7 \mathrm{~mm}$ ventral to the skull surface) or the ventral striatum (the shell of the nucleus accumbens, 1.6 $\mathrm{mm}$ anterior to bregma, $1.0 \mathrm{~mm}$ mediolateral, $4.0 \mathrm{~mm}$ ventral to the skull surface). One week after recovery, subjects returned to selfadministration training, and microinjections began when the acquisition or the reacquisition of a stable response was completed. Fifteen minutes before the self-administration test session, drug or vehicle was injected via an internal cannula extending 0.5 or $3.0 \mathrm{~mm}$ beyond the tip of the guide cannula in the dorsal and ventral striata. The volume infused with a Hamilton $25 \mu \mathrm{l}$ syringe (1702) was $1 \mu \mathrm{l} / \mathrm{side}$ at a rate of $0.5 \mu \mathrm{l} / \mathrm{min}$, with the injectors kept in position in the cannulas for an additional $2 \mathrm{~min}$. All subjects received each drug in a counterbalanced manner. Subjects received one injection per week. The doses used were $0.3,1.5$, and $3 \mathrm{ng} / \mu \mathrm{l}$ $(1,5$, and $10 \mu \mathrm{M})$ for PP2, $1.5 \mathrm{ng} / \mu \mathrm{l}(5 \mu \mathrm{M})$ for PP3, and $0.5 \mu \mathrm{g} / \mu \mathrm{l}$ for ifenprodil. PP2, PP3, and ifenprodil were initially dissolved in DMSO and brought to final concentrations with PBS (final concentration of DMSO, $<1 \%)$. Injection time and doses for PP2 and ifenprodil were based on previous studies (Rodrigues et al., 2001; Bain et al., 2003; Bevilaqua et al., 2005). At the end of the experiments, rats' brains were collected and $75-\mu \mathrm{m}$-thick slices made to check placement of cannulas. Only data from subjects with cannulas located in the region of interest were included in the analysis.

\section{Statistical analysis}

Biochemistry. Data are expressed as mean $\pm \mathrm{SD}$ and analyzed using onesample $t$ test.

Electrophysiology. All data are expressed as mean \pm SEM, and statistical analyses were performed using one-way ANOVA unless otherwise stated.

Behavior. Data were analyzed by one- or two-way ANOVA with repeated measures, followed by the Student-Newman-Keuls test when indicated. Because the latency data to the first press and the first reward did not conform to a normal distribution, the analyses were conducted with the Friedman repeated-measures ANOVA on ranks.

\section{Results}

\section{Exposure of dorsal striatal neurons to ethanol results in NR2B phosphorylation and Fyn activation}

We first examined the phosphorylation state of the NR2A and NR2B subunits in dorsal striatal slices treated with and without ethanol. We observed that acute treatment of slices with ethanol increased tyrosine phosphorylation of the NR2B (Fig. 1A) but not NR2A (Fig. $1 B$ ). Interestingly, the increase in NR2B phosphorylation was specific to the dorsal part of the striatum, because no change in the phosphorylation state of either subunit was observed in ventral striatal slices treated with ethanol (Fig. $1 A, B)$. We next tested whether the increase in NR2B phosphorylation in response to ethanol exposure corresponds with increased activity of Fyn and/or Src, the kinases responsible for phosphorylating the NR2 subunits (Salter and Kalia, 2004). Fyn and Src are fully activated by autophosphorylation of tyrosine 417/418, respectively (Salter and Kalia, 2004), and thus we measured kinase activity by detecting the phosphorylation levels at this site. We found that acute exposure of the dorsal striatum to ethanol results in increased activity of Fyn ex vivo (Fig. 1C) and in vivo (Fig. 1D). This increase in Fyn activity was observed in the dorsal but not ventral striatum (Fig. 1C). However, no change in the activity of Src was detected in the absence or presence of ethanol in either brain region (Fig. 1E). Together, these data suggest that ethanol treatment results in a brain region-specific activation of a pathway that includes Fyn kinase and its substrate, the NR2B subunit of the NMDAR.

Next, we set out to identify a mechanism that may account, at least in part, for the brain region-specific actions of ethanol. Previously, we found that compartmentalization of Fyn to the NR2B subunit by the inhibitory scaffolding protein RACK1 can be detected in the hippocampus but not in the cortex (Yaka et al., 2002, 2003b). We further showed that the localization of Fyn in close proximity to, or away from, the NMDAR is responsible for the difference in the sensitivity of the NMDAR to ethanol in these brain regions (Yaka et al., 2003b). We, therefore, hypothesized that the subregion-specific association of Fyn with the NR2B subunit could be the molecular mechanism underlying the differences in the action of ethanol on the phosphorylation state of the NR2B subunit in the dorsal, but not ventral, striatum. To test this possibility, RACK1 was immunoprecipitated from the two subregions of the striatum, and the levels of coimmunoprecipitated Fyn and NR2B were determined. As shown in Figure $1 F$, Fyn and NR2B coimmunoprecipitated with anti-RACK1 antibodies in the dorsal but not the ventral striatum. Furthermore, as previously reported in the hippocampus (Yaka et al., 2003b), ethanol treatment resulted in the dissociation of Fyn from RACK1 (Fig. $1 F$ ), enabling the kinase to phosphorylate the channel subunit. These results imply that the localization of Fyn near the NR2B subunit in the dorsal but not the ventral striatum underlies, at least in part, the actions of ethanol on the channel. 
A

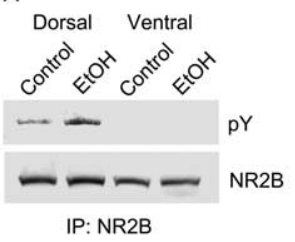

B

C

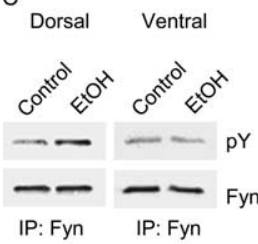

E

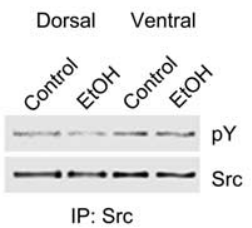

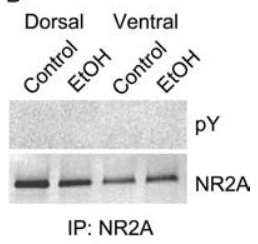

$\mathrm{D}$
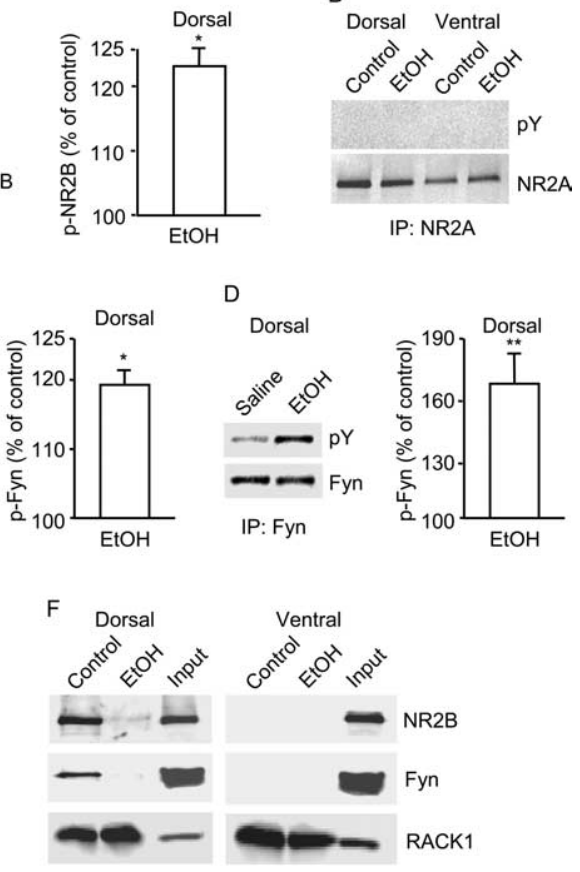

IP: RACK 1

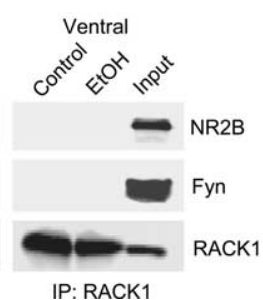

Figure 1. Ethanol treatment increases NR2B subunit phosphorylation and Fyn activation in the rat dorsal striatum. $\boldsymbol{A}$, Left, Ethanol incubation increased the phosphorylation level of the NR2B subunit in the dorsal, but not the ventral, striatum. Dorsal or ventral striatal slices were treated with $100 \mathrm{~mm}$ ethanol for $30 \mathrm{~min}$. Phosphorylation of the NR2B subunit was detected by anti-[pY1252]NR2B antibodies (pY; top). The membrane was stripped and reprobed with antiNR2B antibodies (bottom). The image is representative of five experiments. Right, Bar graph showing ethanol-mediated phosphorylation of the NR2B subunit in the dorsal striatum. The level of [pY1252]NR2B ( $p$-NR2B) was normalized to total NR2B. ${ }^{*} p<0.05$ versus control; $n=$ 5 experiments, two rats per experiment. $\boldsymbol{B}$, Ethanol incubation did not alter the phosphorylation state of the NR2A subunit in the dorsal or the ventral striatum. Dorsal or ventral striatal slices were treated as in $\boldsymbol{A}$. The NR2A subunit was immunoprecipitated with anti-NR2A antibodies, and phosphorylation of NR2A was detected with anti-phosphotyrosine antibodies (pY; top). The membrane was stripped and reprobed with anti-NR2A antibodies (bottom). The image is representative of three independent experiments. $\boldsymbol{C}$, Left, Ethanol treatment increased the activity of Fyn kinase in the dorsal but not the ventral striatum. Dorsal or ventral striatal slices were treated with $100 \mathrm{~mm}$ ethanol for $20 \mathrm{~min}$. Fyn was immunoprecipitated with anti-Fyn antibodies, and activation of Fyn was detected by [pY417] Fyn antibodies (pY; top). The blot was stripped and reprobed with anti-Fyn antibodies (bottom). The image is representative of four independent experiments. Right, Quantification of the level of [pY417]Fyn (p-Fyn) to total immunoprecipitated Fyn. ${ }^{*} p<0.05$ versus control; $n=4$ experiments, 2 rats per experiment. $\boldsymbol{D}$, Left, Systemic administration of ethanol increased the activity of Fyn kinase in the dorsal striatum. C57BL/ 6 mice received three intraperitoneal injections of saline for habituation before test injection of saline or ethanol $(2.5 \mathrm{~g} / \mathrm{kg})$. Fifteen minutes after test injection, the dorsal striatum was dissected and homogenized, and activation of Fyn was detected by [pY417]Fyn antibodies (pY; top). The blot was stripped and reprobed with anti-Fyn antibodies (bottom). The image is representative of six saline and six ethanol injections. Right, Quantification of the level of [pY417]Fyn ( $p$-Fyn) to total immunoprecipitated Fyn. ${ }^{* *} p<0.01$ versus the saline group by Student's test; $n=6$ experiments. $\boldsymbol{E}$, Ethanol does not affect the activity of Srckinase. Dorsal or ventral striatal slices were treated with ethanol as in $\boldsymbol{C}$. The slices were homogenized, and Src was immunoprecipitated. Activation of Src was detected by [pY418]Src antibodies (pY; top). The blot was stripped and reprobed with anti-Src antibodies (bottom). The image is representative of four experiments. $\boldsymbol{F}$, Ethanol modulates the association of RACK1, Fyn, and the NR2B subunit in the dorsal striatum. Dorsal or ventral striatal slices were treated with or without $100 \mathrm{~mm}$ ethanol for $30 \mathrm{~min}$. The slices were homogenized and RACK1 was immunoprecipitated with anti-RACK1 antibodies (bottom), and coimmunoprecipitation was detected with anti-Fyn (middle) and anti-NR2B (top) antibodies. The input lane shows level of proteins in total lysates. The image is representative of three independent experiments.
LTF of NMDAR-mediated synaptic transmission is induced after acute ethanol application in the dorsal but not the ventral striatum

Fyn-mediated phosphorylation of the NR2B subunit of the NMDAR leads to enhanced activity of the channel (Salter and Kalia, 2004). Therefore, we tested whether Fyn activation and NR2B phosphorylation in response to ethanol in the dorsal striatum alter NMDAR-mediated synaptic transmission. Bath application of ethanol resulted in a decrease in NMDAR-mediated EPSCs (NMDAR EPSCs) (Fig. 2A). This result is consistent with our previous studies showing that ethanol exerts an immediate depressive effect on NMDAR activity (Lovinger et al., 1989, 1990). However, after ethanol washout, the NMDAR EPSCs gradually recovered and subsequently increased above baseline (Fig. 2A). This facilitation of NMDAR-mediated activity lasted for $>30 \mathrm{~min}$ (Fig. 2A), and is thus named LTF. Together, these results show that LTF of NMDAR-mediated synaptic transmission is induced after acute ethanol application in the dorsal striatum.

Next, we examined the action of ethanol on NMDAR EPSCs in the ventral striatum, a brain region in which we did not observe Fyn activation or NR2B phosphorylation in response to ethanol (Fig. 1A,C). We found that, similar to the dorsal striatum, the amplitude of NMDAR EPSC was reduced in the presence of ethanol; however, ethanol washout failed to induce LTF of the NMDAR EPSC (Fig. 2 B). Finally, as shown in Figure 2, $C$ and $D$, Fyn activation and NR2B subunit phosphorylation in the dorsal but not ventral striatum were also detected after ethanol washout.

\section{Ethanol-induced LTF in the dorsal striatum is mediated by NR2B-NMDARs and requires Fyn}

The results above suggest that ethanol-induced long-lasting enhancement of channel activity in the dorsal striatum is attributable to Fyn-mediated phosphorylation of the NR2B subunit. If so, then the NR2B-containing NMDAR should mediate the induction of LTF of channel activity in response to ethanol. As expected, we found that the selective NR2B-NMDAR inhibitor, Ro 25-6981, abolished LTF induction (Fig. 3A). In addition, perfusion of Ro 25-6981 resulted in the inhibition of ethanolmediated LTF when applied after the activity of the channel had been enhanced by ethanol washout (Fig. 3B). To further confirm the conclusion that the LTF of NMDAR activity induced by ethanol exposure in the dorsal striatum is mediated by the NR2BNMDAR, we reasoned the following; if LTF is mediated exclusively by the NR2B-NMDAR, but not by other NR2 subunitcontaining NMDARs (non-NR2B-NMDARs), then two predictions can be made: first, the amplitude of EPSCs in the presence of Ro 25-6981 (non-NR2B-NMDAR EPSCs) will be the same in recordings during the LTF period and without ethanol application; second, the ratio of NR2B-/non-NR2B-NMDAR EPSCs will be increased during the LTF of channel activity compared with the ratio before ethanol application. As expected, the amplitude of non-NR2B-NMDAR EPSCs did not change during the LTF of channel activity; however, the ratio of NR2B-/nonNR2B-NMDAR EPSCs was increased (Fig. 3B, bar graph). Together, these results suggest that the amplitude of NR2Bcontaining, but not non-NR2B-containing, NMDAR EPSCs is increased after ethanol washout.

Next, we hypothesized that Fyn-mediated phosphorylation of NR2B is required for the development of LTF in response to ethanol. Therefore, we examined the contribution of Fyn to ethanol-mediated induction of LTF of NMDAR activity by testing the specific inhibitor of the Src family of PTKs, PP2. PP2 did 

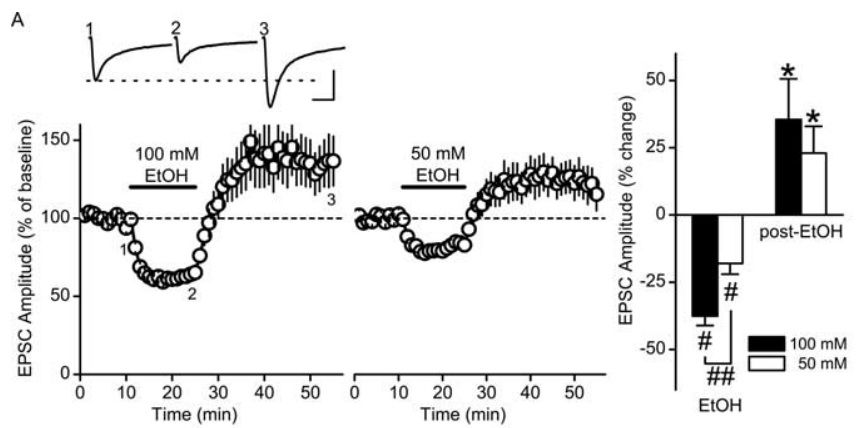

B
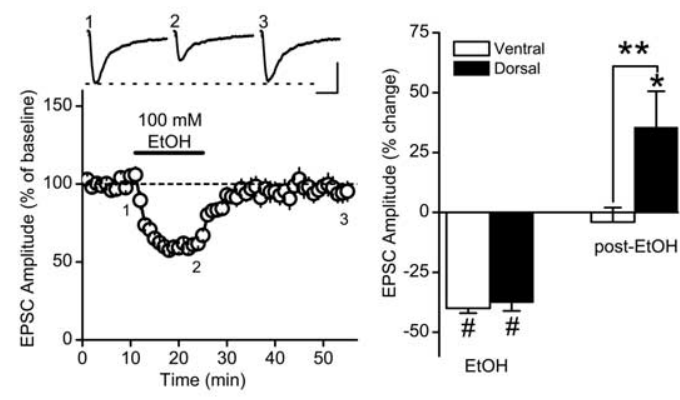

C

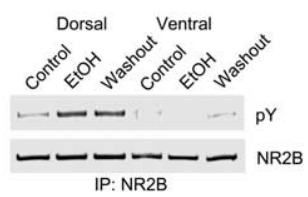

D

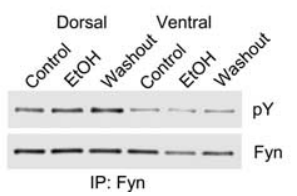

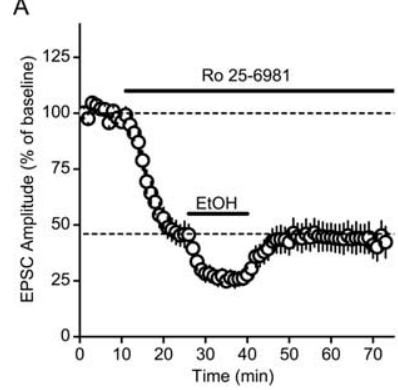

B

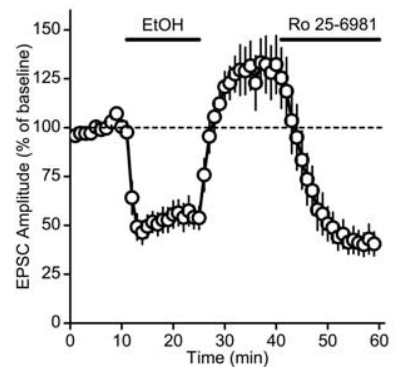

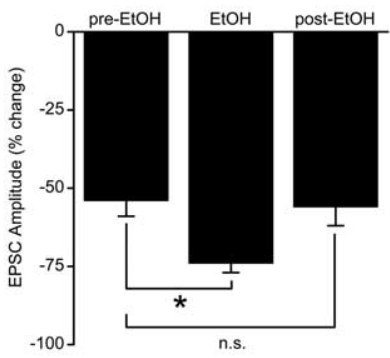

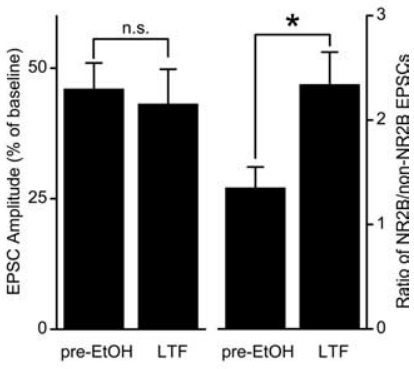

Figure 3. LTF in the rat dorsal striatum is mediated by NR2B-NMDARs. $A$, Blockade of NR2BNMDARs prevents LTF induction by ethanol. Left, Time course of NMDAR EPSCs in the absence and presence of the NR2B antagonist, Ro 25-6981 (0.5 $\mu \mathrm{M})$, and ethanol (100 mM). Note that LTF was not observed after ethanol washout in the presence of Ro 25-6981 ( $n=9$ slices). Right, Bar graph showing the mean NMDAR EPSCs before (pre-EtOH), during (EtOH), and after (postEtOH) $100 \mathrm{~mm}$ ethanol application in the presence of Ro 25-6981. EPSCs were averaged from 13-15 min after starting perfusion of Ro 25-6981 for "pre-EtOH," from 10 to 14 min after starting perfusion of EtOH for "EtOH," and from 21 to 30 min after ethanol washout for "postEtOH." * $p<0.05$; n.s., not significant, $p>0.05$ by paired Student's $t$ test. Error bars indicate SEM. $\boldsymbol{B}$, Ro 25-6981 blocks ethanol-induced LTF of NMDAR EPSCs. Left, time course. $n=10$ slices. Right, Left, Bar graph comparison of EPSCs in the presence of Ro 25-6981 before ethanol application ("pre-EtOH"; quantification of the mean EPSCs 13-15 min after starting perfusion of Ro 25-6981 in $\boldsymbol{A}$, left) and after LTF is established ("LTF"; quantification of the mean EPSCS 13-15 min after starting perfusion of Ro 25-6981 in $\boldsymbol{B}$, left). Note that the EPSCs in the presence of Ro 25-6981 before ethanol application and after LTF is established are not significantly different $(p>0.05)$. Right, Right, Bar graph comparison of the ratios of NR2B to non-NR2B EPSCs before ethanol application and after LTF is established. The non-NR2B EPSC was defined as the EPSC in the presence of Ro 25-6981, whereas the NR2B EPSC was calculated as the difference between the EPSCs before and after Ro 25-6981 application. Note that the ratio of NR2B/non-NR2B EPSCs is increased in LTF compared with pre-EtOH. * $p<0.05$.

Right, Bar graph comparing the direct $(\mathrm{EtOH})$ and washout (post-EtOH) effects of different concentrations of ethanol on NMDAR EPSCs. EPSCs were averaged from 10-14 min after ethanol application (EtOH), and from 21-30 min after ethanol washout (post-EtOH). " $p<0.01$, ${ }^{*} p<0.05$ versus baseline (average of EPSCs over the 10 min before ethanol application) by paired Student's $t$ test; ${ }^{\# \#} p<0.05$. B , Ethanol failed to induce LTF in the ventral striatum. Left, Time course of NMDAR EPSCs recorded in the ventral striatum before, during, and after $100 \mathrm{~mm}$ ethanol application. Note that, on average, no LTF was observed ( $n=14$ slices). Right, Bar graph comparing the ethanol (100 mm) effect on mean NMDAR EPSCs in the dorsal (same data as $\boldsymbol{A}$, left) and the ventral (same data as $\boldsymbol{B}$, left) striatum. ${ }^{\#} p<0.01,{ }^{*} p<0.05$ versus baseline; ${ }^{* *} p<0.05$. C, Sustained NR2B subunit phosphorylation after ethanol washout in the dorsal striatum. Dorsal or ventral striatal slices were treated with $100 \mathrm{~mm}$ ethanol for $20 \mathrm{~min}$. The slices were homogenized and the NR2B subunit was immunoprecipitated. Phosphorylation of the NR2B subunit was detected by anti-[pY1252]NR2B antibodies (pY; top). The membrane was stripped and reprobed with anti-NR2B antibodies (bottom). The image is representative of three independent experiments. Quantification of the level of pYNR2B ( $p$-NR2B) to total immunoprecipitated NR2B subunits is shown. ${ }^{*} p<0.05,{ }^{* *} p<0.01$ versus control; $n=3$ experiments, two rats per experiment. $D$, Sustained activation of Fyn activity after ethanol washout in the dorsal striatum. Dorsal or ventral striatal slices were treated with $100 \mathrm{~mm}$ ethanol for $15 \mathrm{~min}$. Ethanol was removed, and fresh aCSF was added for $10 \mathrm{~min}$. The slices were homogenized, and Fyn was immunoprecipitated with anti-Fyn antibodies. Activation of Fyn was detected by anti[pY417]Fyn antibodies (pY; top). The blot was stripped and reprobed with anti-Fyn antibodies (bottom). The image is representative of four independent experiments. Quantification of the level of [pY417]Fyn (p-Fyn) to total immunoprecipitated Fyn is shown. ${ }^{*} p<0.05,{ }^{* *} p<0.01$ versus control; $n=4$ experiments, two rats per experiment.

not alter the magnitude (Fig. $4 A, C$ ), or the time course of acute ethanol inhibition of channel activity (Fig. 4A). However, no LTF was observed after ethanol exposure in slices treated with PP2 (Fig. 4A, C). To confirm that Fyn kinase is required for the induction of the prolonged enhancement of NMDAR activity in the dorsal striatum in response to ethanol, we compared the ethanolmediated induction of LTF in Fyn knock-out mice $\left(\mathrm{Fyn}^{-1-}\right)$ and their wild-type littermates $\left(\mathrm{Fyn}^{+/+}\right)$. Similar to the results obtained from rat dorsal striatal slices (Fig. 2A), we observed LTF after ethanol exposure in the dorsal striatum of $\mathrm{Fyn}^{+/+}$mice; however, no LTF was detected in Fyn ${ }^{-1-}$ mice (Fig. $4 B, C$ ). These results suggest that Fyn kinase is required for ethanol-mediated induction of LTF in the dorsal striatum.

LTF of NMDAR-mediated synaptic transmission in the dorsal striatum is not accompanied by changes in the probability of transmitter release

Our results thus far suggest a postsynaptic mechanism for the enhancement of NMDAR activity in response to ethanol. To determine whether the facilitation of NMDAR EPSCs in the dorsal striatum also involves a presynaptic component, we examined 

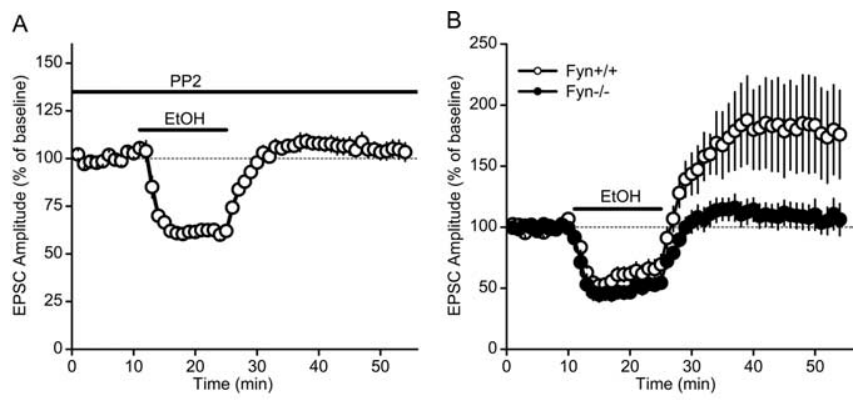

C

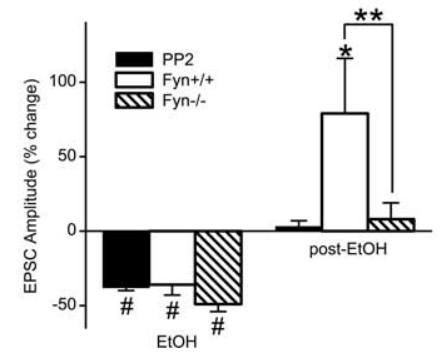

Figure 4. Fyn kinase is required for ethanol-induced LTF in the dorsal striatum. $\boldsymbol{A}$, The Src family antagonist, PP2, prevents LTF induction by ethanol in the rat dorsal striatum. PP2 $(1 \mu \mathrm{m})$ was bath-applied and present throughout the recordings. Note that LTF was not detected after ethanol washout ( $n=11$ slices). $\boldsymbol{B}$, Deletion of the Fyn kinase gene prevents ethanol-induced LTF in the mouse dorsal striatum. Note that the LTF induced by ethanol was observed in Fyn ${ }^{+/+}$mice $(n=12$ slices $)$ but not in Fyn ${ }^{-1-}$ mice $(n=16$ slices $)$. C, Summary of the mean NMDAR EPSCs during (EtOH) and after (post-EtOH) ethanol applications in $\boldsymbol{A}$ and $\boldsymbol{B}$ is shown. ${ }^{\#} p<0.01,{ }^{*} p<0.05$ versus corresponding baselines; ${ }^{* *} p<0.05$. Error bars indicate SEM.

paired-pulse (100 ms interpulse interval) ratios (PPRs) (Zucker and Regehr, 2002) of NMDAR EPSCs before ethanol application and after LTF had been established. There was no difference between PPRs of NMDAR EPSCs before ethanol application and after LTF was established (Fig. 5A3,A4,B). This result suggests that the probability of transmitter release is not altered during LTF of NMDAR EPSCs in the dorsal striatum. To further confirm this conclusion, we compared PPRs of AMPAR-mediated EPSCs before and after ethanol application. We found that there was no significant difference between PPRs of AMPAR EPSCs before and after ethanol application (Fig. 5C,D). In addition, ethanol treatment did not induce LTF of AMPAR EPSCs after washout of acute ethanol application (the mean amplitude of AMPAR EPSCs $21-30 \mathrm{~min}$ after ethanol washout is $95.9 \pm 13.0 \%$ of baseline; $p>0.05 ; n=6$ slices). Therefore, an increase in the probability of glutamate release does not contribute to the long-term enhancement of NMDAR activity after ethanol washout.

\section{Dorsal striatal inhibition of Src family PTKs or NR2B-} NMDAR attenuates operant ethanol self-administration Finally, we investigated possible behavioral consequences of Fynmediated long-lasting enhancement of NR2B-NMDAR activity. To do so, we microinjected the Src family PTKs inhibitor PP2 and the NR2B-NMDAR antagonist ifenprodil into the dorsal striatum of rats trained to self-administer ethanol. We found that intradorsal striatal infusion of PP2, but not its inactive analog PP3, significantly reduced operant self-administration of ethanol (Fig. 6A). Similarly, inhibition of NR2B-NMDARs in the dorsal striatum with ifenprodil attenuated ethanol self-administration (Fig. 6A).

We further analyzed the data by assessing the mean distribution of active lever press responding as a function of session time after each treatment. The latencies to the first press and to the first reward after PP2 and ifenprodil treatments were not different from that of the first press after vehicle administration $(p=0.87$ and 0.77 by the Friedman test for PP2 and ifenprodil, respectively) (data not shown). Additional analysis of cumulative active lever press responding within the test session showed that the decreases in responding induced by PP2 and ifenprodil treatment are not significant until 15-20 min after the beginning of the session (supplemental Fig. 2, available at www.jneurosci.org as supplemental material). Hence, PP2 and ifenprodil do not alter initiation of ethanol self-administration; the decrease in behavior occurs after a period of reinforced responding. We also found that the distribution of interresponse intervals was similar for vehicle, PP2 (1.5 ng) and ifenprodil (no effect of treatment, $F_{(3,599)}=0.73, p=0.54$; and no interaction between treatment and time interval, $F_{(60,599)}=0.93, p=0.62$ ) (Fig. $6 B$ ). Most importantly, no change in the proportion of fast responses (interresponse intervals of $<1.0 \mathrm{~s}$ ) was observed ( $p$ values $>0.355$ ). These results indicate that the effects of intradorsal striatal infusion of PP2 or ifenprodil on operant self-administration of ethanol are not attributable to inhibition of locomotor activity. To determine the specificity of PP2 and the effects of ifenprodil, we examined their ability to attenuate self-administration of another rewarding substrate, sucrose. As shown in Figure 6C, intradorsal striatal microinjection of PP2 or ifenprodil did not alter selfadministration of sucrose. Finally, we determined whether an effective dose of PP2 and ifenprodil microinjected into the ventral striatum attenuates ethanol self-administration. The ventral striatum is a brain region in which Fyn activity and NR2B subunit phosphorylation were not altered in response to ethanol, and LTF was not observed. As shown in Figure $6 D$, intraventral striatal infusion of PP2 or ifenprodil did not affect operant selfadministration of ethanol.

\section{Discussion}

Here, we present data to suggest that the tyrosine kinase Fyn and its substrate the NR2B subunit of the NMDAR are part of a signaling pathway that is specifically activated in the dorsal striatum during ethanol exposure and contributes to the molecular mechanisms underlying the maintenance of ethanol selfadministration. Specifically, we show that exposure of dorsal striatal neurons to ethanol leads to Fyn activation and phosphorylation of the NR2B subunit. We further provide evidence to suggest that Fyn-mediated phosphorylation of NR2B results in LTF of NR2B-NMDAR EPSCs. Finally, we show that inhibition of Src family tyrosine kinases and NR2B-containing NMDARs attenuates operant self-administration of ethanol.

An important finding that stems from our study is the specificity of the actions of ethanol. We observed that Fyn, but not its highly homologous family member Src, was activated in response to ethanol. This led to the specific phosphorylation of the NR2B, but not the NR2A, subunit of the NMDAR, and to the prolonged enhancement of NR2B-NMDAR. Importantly, none of these effects were observed in the structurally related ventral striatum. Our data suggest that the molecular difference in response to ethanol between these two striatal subregions may be attributable to the differences in the compartmentalization of Fyn near (dorsal striatum) or away from (ventral striatum) the NMDAR complex. Signaling differences in brain regions have been previously reported. For instance, differences in the cholesterol levels in the cortex and hippocampus are suggested to underlie greater outgrowth in cortical neurons than in hippocampal neurons (Ko et al., 2005), and we previously reported that the compartmentalization of Fyn underlies, at least in part, the differences in the 
A
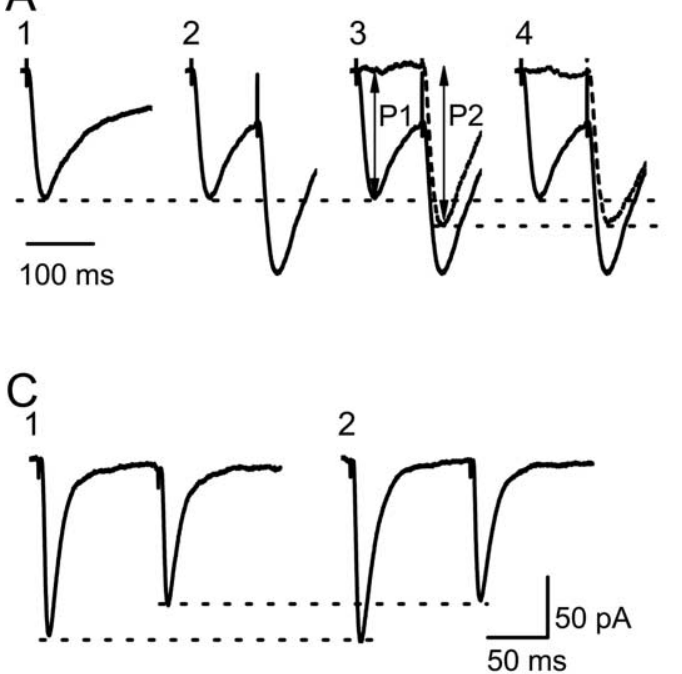

$\mathrm{B}$
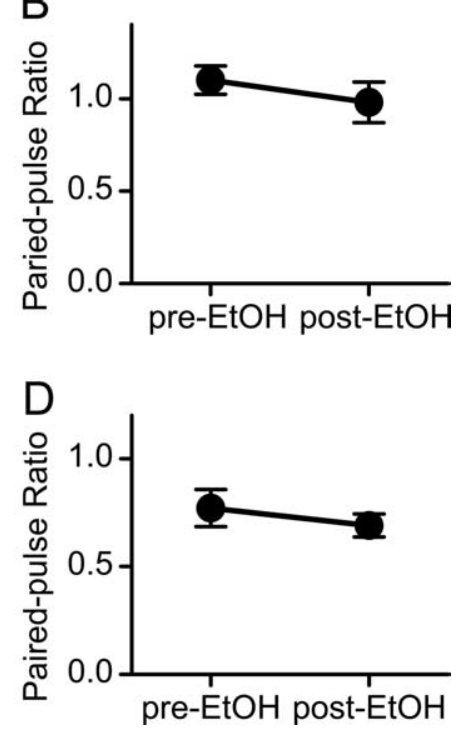

Figure 5. Probability of neurotransmitter release remains unchanged during ethanol-induced LTF. $\boldsymbol{A}$, Sample traces of the paired-pulse NMDAR EPSCs obtained before ethanol application (3, solid line) and after LTF was established (4, solid line) and explanation of how the PPR of the EPSCs was calculated (1-3). 1, A single EPSC elicited by one stimulus; 2, paired-pulse EPSCs elicited by two stimuli with an interval of $100 \mathrm{~ms}$. Single EPSCs and paired-pulse EPSCs were alternately induced at an interval of $20 \mathrm{~s} ; 3$, the true amplitude of the second EPSC (dashed line) can be seen after digital subtraction of the single EPSC (1) from the paired-pulse EPSCs (2). The PPR of NMDAREPSCs was defined as the ratio of P2 over P1, where P2 and P1 are the amplitudes of the subtracted second EPSC (dashed line) and the first EPSC in the paired-pulse EPSCs (solid line), respectively. 3 also shows unsubtracted sample traces obtained before ethanol application and $\mathbf{4}$ after LTF was established. $\boldsymbol{B}$, Line graph summarizing PPRs of NMDAR EPSCs obtained before ethanol application (pre-EtOH) and after LTF is established (post-EtOH). PPRs are not significantly different ( $p>0.05$ by paired Student's $t$ test; $n=7$ slices). Error bars indicate SEM. C, Sample traces of the paired-pulse AMPAR EPSCs obtained before ethanol application (1) and after LTF was established (2). D, Line graph summarizing the PPRs of AMPAR EPSCs obtained before and after ethanol application. The PPRs between pre- and post-EtOH applications are not significantly different ( $p>0.05$ by paired Student's $t$ test; $n=6$ slices).

actions of ethanol in the hippocampus versus the cortex (Yaka et al., 2003b). Some differences are also found between the dorsal and the ventral striatum. For example, chronic morphine administration increases the activity of extracellular signal-regulated protein kinases (ERKs) in the dorsal but not the ventral striatum, although the level of total ERKs is higher in the ventral than in the dorsal striatum (Ortiz et al., 1995). In addition, increased $\mathrm{Ca}^{2+}$ influx through NMDARs and L-type $\mathrm{Ca}^{2+}$ channels leads to robust cAMP response element-binding protein (CREB) phosphorylation in the dorsal striatum, but only to low or moderate CREB phosphorylation in the ventral striatum (Liu and Graybiel, 1998). Finally, the acute effects of dopamine on glutamatergic transmission are different in the dorsal versus the ventral striatum. In the ventral striatum, dopamine inhibits such transmission at low frequencies of afferent activation via activation of the dopamine $\mathrm{D}_{1}$ receptor (Nicola et al., 2000), whereas in the dorsal striatum, inhibition involves the dopamine $\mathrm{D}_{2}$ receptors, and is mainly observed at higher frequencies of afferent activation (Bamford et al., 2004; Yin and Lovinger, 2006).

We observed a long-term enhancement of NR2B-NMDAR activity after ethanol washout. This result is consistent with other long-lasting effects of ethanol on NMDARs reported in acute spinal cord slices ( $\mathrm{Li}$ et al., 2005). This finding also resembles the enhancement in NMDAR-mediated currents we recently observed in the ventral tegmental area after washout of cocaine (Schilstrom et al., 2006). However, we previously observed that, in the hippocampus, ethanol washout resulted in a rebound potentiation of NMDAR activity that rapidly decayed to baseline (Yaka et al., 2003b). This difference might be attributable to the fact that the NR2B subunit is the primary NMDAR subunit in the dorsal striatum, whereas hippocampal neurons express a greater proportion of non-NR2BNMDARs (Kawakami et al., 2003). It is also plausible, however, that other, yet unidentified molecular differences, such as differences in expression and/or activity of tyrosine phosphatases that regulate the phosphorylation state and activity of Fyn and the NR2 subunits (Braithwaite et al., 2006), underlie the variation in the decay time of NMDAR activity after ethanol washout in these brain regions.

Our results suggest that Fyn-mediated NR2B subunit phosphorylation underlies the LTF in NMDAR activity in response to ethanol. How does tyrosine phosphorylation of NMDARs induced by ethanol facilitate NMDAR activity? First, tyrosine phosphorylation of NMDARs is reported to enhance gating of NMDAR channels (Salter and Kalia, 2004). Second, tyrosine phosphorylation of NMDARs induced by ethanol may facilitate the trafficking of NMDARs from the internal pool to the cell membrane, thereby increasing NMDAR function (Dunah et al., 2004). Finally, it is possible that the initial increase in channel activity requires Fyn, whereas the maintenance of NMDAR EPSC facilitation may be mediated by other signaling pathways, such as autonomous activation of enzymes like the CaM kinase II (calciumdependent calcium calmodulin kinase II) (Bayer et al., 2001).

An important implication of our current findings is that facilitation of NMDAR activity by ethanol may produce aberrant synaptic plasticity in the dorsal striatum. The long-term potentiation of AMPAR-mediated synaptic transmission (AMPAR LTP) in the dorsal striatum is reported to be NMDAR dependent $(\mathrm{Ca}-$ labresi et al., 1992; Partridge et al., 2000; Kerr and Wickens, 2001), and the NR2B-NMDARs have been reported to be involved in AMPAR LTP in the hippocampus (Barria and Malinow, 2005). Therefore, it will be of great interest to determine whether LTF of NMDAR activity by ethanol facilitates the induction of AMPAR LTP in the dorsal striatum.

Importantly, we present evidence to suggest that the same signaling pathway in the dorsal striatum responsible for LTF of NMDAR EPSCs also contributes to the mechanism that underlies operant self-administration of ethanol. Although PP2 inhibits all members of the Src PTKs, and we cannot exclude the possibility that the behavior was mediated by another Src PTK, this is unlikely because we did not detect activation of Src in the dorsal striatum on ethanol exposure, and moreover, our previous study suggested that Src activity is inhibited in the presence of ethanol (Suvarna et al., 2005). Furthermore, the LTF of NR2B-NMDAR activity was abolished in the Fyn ${ }^{-1-}$ mice, suggesting that Fyn is necessary for the process. Finally, inhibition of Src PTKs or NR2B-NMDARs did not alter operant self-administration of ethanol when the inhibitors were injected into the ventral striatum, suggesting a specific contribution of the dorsal striatum to ethanol drinking behavior via Fyn and NR2B-NMDAR.

The dorsal striatum is responsible for movement control 
(Graybiel et al., 1994); however, it is unlikely that the decrease in ethanol selfadministration can be explained by changes in locomotor activity. First, there was no effect of PP2 or ifenprodil on the latencies to the first press and to the first reward. Second, we found no effect of PP2 or ifenprodil on the interresponse interval distribution, indicating that the local response rates were not changed by the treatments. Third, effective doses of PP2 and ifenprodil did not alter self-administration of a natural reward (i.e., sucrose). Hence, PP2- and ifenprodil-treated subjects were not impaired in the initiation and production of lever press responding. It is possible that infusion of PP2 or infenprodil into the dorsal striatum reduces operant ethanol self-administration via one or both of the following mechanisms. First, it is possible that the training process of operant ethanol self-administration (60 min a day and $5 \mathrm{~d}$ a week for 2 months) increases the basal level of Fyn and NR2B-NMDAR activities. Such a sustained change in Fyn activity in turn may lead to a higher basal level of NR2B phosphorylation and PP2 and ifenprodil therefore may inhibit the increased basal level of NR2B-NMDAR activity. To test this possibility, we measured the effect of PP2 on basal level of NMDAR EPSCs and after ethanolmediated LTF of NMDAR activity was established. We found that PP2 did not alter basal NMDAR EPSCs within $30 \mathrm{~min}$ (data not shown). This result is consistent with previous studies (Kim et al., 2002; Lin et al., 2003; Yaka, 2003a). However, we found that the average NMDAR EPSC was slightly decreased starting 10 min after PP2 application (data not shown). A one-way ANOVA conducted on the time after PP2 application showed a marginal effect $\left(F_{(7,203)}=1.48 ; p=0.06 ; n=8\right)$. Together, these results suggest that PP2 might decrease channel function when the activity has already been elevated. However, because the reduction of NMDAR EPSCs when PP2 was applied after the establishment of LTF was only partial, it is possible that Fyn is essential for the initiation of LTF but less for its maintenance. Second, it is possible that the infusion of PP2 or ifenprodil attenuates operant self-administration of ethanol by preventing ethanol-mediated LTF induction in the dorsal striatum. According to this model, in rats infused with vehicle or PP3 infusion, LTF is induced during the test session, and LTF is required for operant self-administration of ethanol. In rats infused with PP2 or ifenprodil, LTF induction is inhibited and operant ethanol self-administration is thus reduced. This possibility is supported by the delayed onset of action of PP2 and ifenprodil during the test session, such that the subject experiences a considerable number of ethanol-reinforced responses before responding diminishes.

It is notable that experimental evidence to support an important role for the dorsal striatum in drug and alcohol-self-
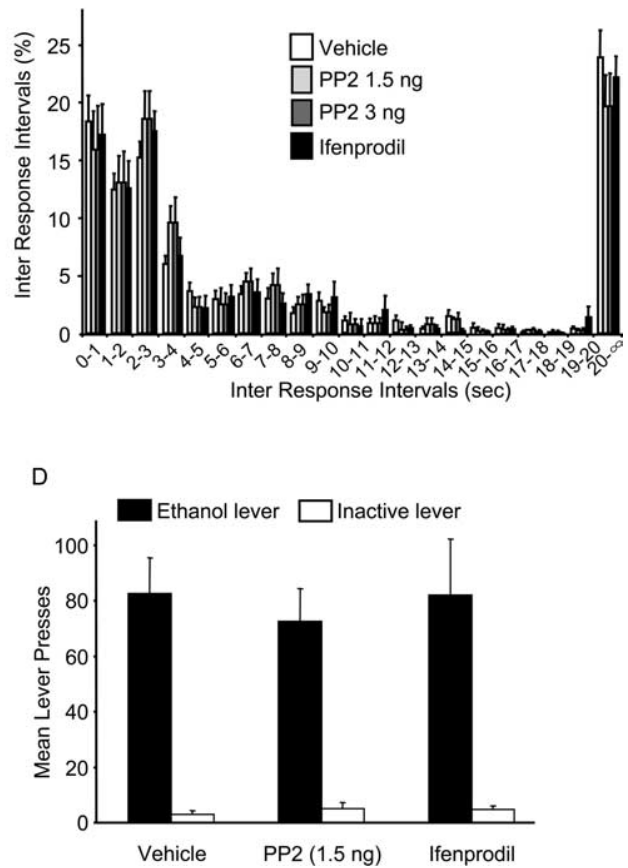

Figure 6. $\quad \boldsymbol{A}$, Intradorsal striatal injections of PP2 and ifenprodil attenuate operant ethanol self-administration. PP2 (0.3, 1.5, and $3 \mathrm{ng}$; equivalent to 1, 5, and $10 \mu \mathrm{m}$, respectively), PP3 (1.5 ng), and ifenprodil ( $0.5 \mu \mathrm{g}$ ) were microinjected ( $1 \mu \mathrm{l} / \mathrm{side})$ into the dorsal striatum 15 min before the beginning of the test session. Data are expressed as mean \pm SEM of number of lever presses second factor) found significant main effects and a significant interaction between both factors ( $F$ values $>4.55 ; p$ values $<$ . Post hoc comparisons revealed significantly reduced active lever responding after 1.5 and $3 \mathrm{ng}$ PP2 and ifenprodil, compared values $>0.89),{ }^{*} p<0.01,{ }^{* *} p<0.001$. Mean ethanol intakes were $0.48 \pm 0.06 \mathrm{~g} / \mathrm{kg}$ for vehicle, $0.46 \pm 0.08,0.31 \pm 0.05$ and $=0.05 \mathrm{~g} / \mathrm{kg}$, respectively, for the doses of $0.3,1.5$, and $3 \mathrm{ng}$ of PP2, $0.46 \pm 0.07$ for $P P 3$, and $0.34 \pm 0.07$ for ifenprodil. $B$ relative number of interresponse intervals (IRIs) (expressed as the percentage of total IRIs) with all intervals $\leq 20$ s presented in , but no effect of treatment and no interaction (Fvalues $<0.93 ; p$ values $>0.54$ ). C, Intradorsal striatal injection of PP2 (1.5 作 $0.59) ; n=14$ rats. $D$, Infusion of an effective dose of PP2 $(1.5 \mathrm{ng})$ or ifenprodil $(0.5 \mu \mathrm{g})$ into the ventral striatum does not reduce operant ethanol self-administration. A two-way ANOVA with repeated measures found a significant effect of lever $\left(F_{(1,12)}=\right.$ $36.67 ; p<.001)$, but no effect of treatment and no interaction ( $F$ values $<0.46 ; p>0.64) ; n=7$ rats.

administration is accruing (McGough et al., 2004; Vanderschuren et al., 2005; Jeanblanc et al., 2006). For example, we recently found that ethanol-induced activation of the BDNF signaling pathway occurs selectively in the dorsal striatum, and may also contribute to the regulation of ethanol consumption (McGough et al., 2004; Jeanblanc et al., 2006). In humans, long-term use of alcohol may lead to development of addictive phenotypes such as repeated, uncontrolled ethanol consumption regardless of the consequences (Kosten and O'Connor, 2003). It has been suggested that prolonged drug experience leads to the development of persistent forms of habit learning (Deroche-Gamonet et al., 2004; Vanderschuren and Everitt, 2004), which most likely depend on the dorsal striatum (Everitt and Robbins, 2005; Vanderschuren et al., 2005; Dang et al., 2006). It is possible therefore that the mechanism described here contributes to the development of such maladaptive habits.

In summary, we have shown that exposure of the dorsal striatum to ethanol leads to LTF of NMDAR activity after ethanol 
washout via Fyn phosphorylation of the NR2B subunit of the NMDAR. Our results further suggest that Fyn and NR2BNMDARs contribute to mechanisms that underlie operant ethanol self-administration. Therefore, this facilitation of the NR2BNMDAR in the dorsal striatum may underlie the rapid adaptive responses of animals and humans to alcohol challenge, and may be an important mechanism leading to the habitual and compulsive alcohol seeking and taking that characterize addiction. Furthermore, a specific inhibitor of Fyn activation in the dorsal striatum could be developed as a drug to treat adverse effects of alcohol exposure, including the development of excessive alcohol intake.

\section{References}

Bain J, McLauchlan H, Elliott M, Cohen P (2003) The specificities of protein kinase inhibitors: an update. Biochem J 371:199-204.

Bamford NS, Zhang H, Schmitz Y, Wu NP, Cepeda C, Levine MS, Schmauss C, Zakharenko SS, Zablow L, Sulzer D (2004) Heterosynaptic dopamine neurotransmission selects sets of corticostriatal terminals. Neuron 42:653-663.

Barria A, Malinow R (2005) NMDA receptor subunit composition controls synaptic plasticity by regulating binding to CaMKII. Neuron 48:289-301.

Bayer KU, De Koninck P, Leonard AS, Hell JW, Schulman H (2001) Interaction with the NMDA receptor locks CaMKII in an active conformation. Nature 411:801-805.

Berke JD, Hyman SE (2000) Addiction, dopamine, and the molecular mechanisms of memory. Neuron 25:515-532.

Bevilaqua LR, da Silva WN, Medina JH, Izquierdo I, Cammarota M (2005) Extinction and reacquisition of a fear-motivated memory require activity of the Src family of tyrosine kinases in the CA1 region of the hippocampus. Pharmacol Biochem Behav 81:139-145.

Bisaga A, Popik P, Bespalov AY, Danysz W (2000) Therapeutic potential of NMDA receptor antagonists in the treatment of alcohol and substance use disorders. Expert Opin Investig Drugs 9:2233-2248.

Braithwaite SP, Paul S, Nairn AC, Lombroso PJ (2006) Synaptic plasticity: one STEP at a time. Trends Neurosci 29:452-458.

Calabresi P, Pisani A, Mercuri NB, Bernardi G (1992) Long-term potentiation in the striatum is unmasked by removing the voltage-dependent magnesium block of NMDA receptor channels. Eur J Neurosci 4:929-935.

Cull-Candy SG, Leszkiewicz DN (2004) Role of distinct NMDA receptor subtypes at central synapses. Sci STKE 2004:re16.

Dang MT, Yokoi F, Yin HH, Lovinger DM, Wang Y, Li Y (2006) Disrupted motor learning and long-term synaptic plasticity in mice lacking NMDAR1 in the striatum. Proc Natl Acad Sci USA 103:15254-15259.

Deroche-Gamonet V, Belin D, Piazza PV (2004) Evidence for addiction-like behavior in the rat. Science 305:1014-1017.

Dunah AW, Sirianni AC, Fienberg AA, Bastia E, Schwarzschild MA, Standaert DG (2004) Dopamine D1-dependent trafficking of striatal $N$-methyl-Daspartate glutamate receptors requires Fyn protein tyrosine kinase but not DARPP-32. Mol Pharmacol 65:121-129.

Everitt BJ, Robbins TW (2005) Neural systems of reinforcement for drug addiction: from actions to habits to compulsion. Nat Neurosci 8:1481-1489.

Fregly MJ, Rowland NE (1992) Comparison of preference thresholds for $\mathrm{NaCl}$ solution in rats of the Sprague-Dawley and Long-Evans strains. Physiol Behav 51:915-918.

Gerdeman GL, Partridge JG, Lupica CR, Lovinger DM (2003) It could be habit forming: drugs of abuse and striatal synaptic plasticity. Trends Neurosci 26:184-192.

Graybiel AM, Aosaki T, Flaherty AW, Kimura M (1994) The basal ganglia and adaptive motor control. Science 265:1826-1831.

Harker KT, Whishaw IQ (2002) Place and matching-to-place spatial learning affected by rat inbreeding (Dark-Agouti, Fischer 344) and albinism (Wistar, Sprague-Dawley) but not domestication (wild rat vs. LongEvans, Fischer-Norway). Behav Brain Res 134:467-477.

Holter SM, Danysz W, Spanagel R (2000) Novel uncompetitive N-methylD-aspartate (NMDA)-receptor antagonist MRZ 2/579 suppresses ethanol intake in long-term ethanol-experienced rats and generalizes to ethanol cue in drug discrimination procedure. J Pharmacol Exp Ther 292:545-552.
Ito R, Dalley JW, Robbins TW, Everitt BJ (2002) Dopamine release in the dorsal striatum during cocaine-seeking behavior under the control of a drug-associated cue. J Neurosci 22:6247-6253.

Jeanblanc J, He DY, McGough NN, Logrip ML, Phamluong K, Janak PH, Ron D (2006) The dopamine D3 receptor is part of a homeostatic pathway regulating ethanol consumption. J Neurosci 26:1457-1464.

Kawakami R, Shinohara Y, Kato Y, Sugiyama H, Shigemoto R, Ito I (2003) Asymmetrical allocation of NMDA receptor $\varepsilon 2$ subunits in hippocampal circuitry. Science 300:990-994.

Kerr JN, Wickens JR (2001) Dopamine D-1/D-5 receptor activation is required for long-term potentiation in the rat neostriatum in vitro. J Neurophysiol 85:117-124.

Khanna JM, Kalant H, Shah G, Sharma H (1990) Comparison of sensitivity and alcohol consumption in four outbred strains of rats. Alcohol $7: 429-434$

Khanna JM, Shah G, Weiner J, Wu PH, Kalant H (1993) Effect of NMDA receptor antagonists on rapid tolerance to ethanol. Eur J Pharmacol 230:23-31.

Kim TY, Hwang JJ, Yun SH, Jung MW, Koh JY (2002) Augmentation by zinc of NMDA receptor-mediated synaptic responses in CA1 of rat hippocampal slices: mediation by Src family tyrosine kinases. Synapse 46:49-56.

Ko M, Zou K, Minagawa H, Yu W, Gong JS, Yanagisawa K, Michikawa M (2005) Cholesterol-mediated neurite outgrowth is differently regulated between cortical and hippocampal neurons. J Biol Chem 280:42759-42765

Kosten TR, O'Connor PG (2003) Management of drug and alcohol withdrawal. N Engl J Med 348:1786-1795.

Krystal JH, Petrakis IL, Mason G, Trevisan L, D'Souza DC (2003) $\mathrm{N}$-Methyl-D-aspartate glutamate receptors and alcoholism: reward, dependence, treatment, and vulnerability. Pharmacol Ther 99:79-94.

Kumari M, Ticku MK (2000) Regulation of NMDA receptors by ethanol. Prog Drug Res 54:152-189.

Li HF, Mochly-Rosen D, Kendig JJ (2005) Protein kinase C $\gamma$ mediates ethanol withdrawal hyper-responsiveness of NMDA receptor currents in spinal cord motor neurons. Br J Pharmacol 144:301-307.

Lin B, Arai AC, Lynch G, Gall CM (2003) Integrins regulate NMDA receptor-mediated synaptic currents. J Neurophysiol 89:2874-2878.

Liu FC, Graybiel AM (1998) Region-dependent dynamics of cAMP response element-binding protein phosphorylation in the basal ganglia. Proc Natl Acad Sci USA 95:4708-4713.

Lovinger DM, White G, Weight FF (1989) Ethanol inhibits NMDAactivated ion current in hippocampal neurons. Science 243:1721-1724.

Lovinger DM, White G, Weight FF (1990) NMDA receptor-mediated synaptic excitation selectively inhibited by ethanol in hippocampal slice from adult rat. J Neurosci 10:1372-1379.

Malenka RC, Nicoll RA (1999) Long-term potentiation-a decade of progress? Science 285:1870-1874.

Martin SJ, Grimwood PD, Morris RG (2000) Synaptic plasticity and memory: an evaluation of the hypothesis. Annu Rev Neurosci 23:649-711.

McGough NN, He DY, Logrip ML, Jeanblanc J, Phamluong K, Luong K, Kharazia V, Janak PH, Ron D (2004) RACK1 and brain-derived neurotrophic factor: a homeostatic pathway that regulates alcohol addiction. J Neurosci 24:10542-10552.

Nicola SM, Surmeier J, Malenka RC (2000) Dopaminergic modulation of neuronal excitability in the striatum and nucleus accumbens. Annu Rev Neurosci 23:185-215.

Ortiz J, Harris HW, Guitart X, Terwilliger RZ, Haycock JW, Nestler EJ (1995) Extracellular signal-regulated protein kinases (ERKs) and ERK kinase (MEK) in brain: regional distribution and regulation by chronic morphine. J Neurosci 15:1285-1297.

Packard MG, Teather LA (1997) Double dissociation of hippocampal and dorsal-striatal memory systems by posttraining intracerebral injections of 2-amino-5-phosphonopentanoic acid. Behav Neurosci 111:543-551.

Partridge JG, Tang KC, Lovinger DM (2000) Regional and postnatal heterogeneity of activity-dependent long-term changes in synaptic efficacy in the dorsal striatum. J Neurophysiol 84:1422-1429.

Prusky GT, Harker KT, Douglas RM, Whishaw IQ (2002) Variation in visual acuity within pigmented, and between pigmented and albino rat strains. Behav Brain Res 136:339-348.

Rodrigues SM, Schafe GE, LeDoux JE (2001) Intra-amygdala blockade of 
the NR2B subunit of the NMDA receptor disrupts the acquisition but not the expression of fear conditioning. J Neurosci 21:6889-6896.

Ron D (2004) Signaling cascades regulating NMDA receptor sensitivity to ethanol. Neuroscientist 10:325-336.

Ronesi J, Gerdeman GL, Lovinger DM (2004) Disruption of endocannabinoid release and striatal long-term depression by postsynaptic blockade of endocannabinoid membrane transport. J Neurosci 24:1673-1679.

Salter MW, Kalia LV (2004) Src kinases: a hub for NMDA receptor regulation. Nat Rev Neurosci 5:317-328.

Schilstrom B, Yaka R, Argilli E, Suvarna N, Schumann J, Chen BT, Carman M, Singh V, Mailliard WS, Ron D, Bonci A (2006) Cocaine enhances NMDA receptor-mediated currents in ventral tegmental area cells via dopamine $\mathrm{D}_{5}$ receptor-dependent redistribution of NMDA receptors. J Neurosci 26:8549-8558.

Sucher NJ, Awobuluyi M, Choi YB, Lipton SA (1996) NMDA receptors: from genes to channels. Trends Pharmacol Sci 17:348-355.

Suvarna N, Borgland SL, Wang J, Phamluong K, Auberson YP, Bonci A, Ron D (2005) Ethanol alters trafficking and functional NMDA receptor NR2 subunit ratio via H-RAS. J Biol Chem 280:31450-31459.

Takasu MA, Dalva MB, Zigmond RE, Greenberg ME (2002) Modulation of NMDA receptor-dependent calcium influx and gene expression through EphB receptors. Science 295:491-495.

Teather LA, Packard MG, Bazan NG (2001) Differential interaction of platelet-activating factor and NMDA receptor function in hippocampal and dorsal striatal memory processes. Neurobiol Learn Mem 75:310-324.

Vanderschuren LJ, Everitt BJ (2004) Drug seeking becomes compulsive after prolonged cocaine self-administration. Science 305:1017-1019.

Vanderschuren LJ, Di Ciano P, Everitt BJ (2005) Involvement of the dorsal striatum in cue-controlled cocaine seeking. J Neurosci 25:8665-8670.
Volkow ND, Wang GJ, Telang F, Fowler JS, Logan J, Childress AR, Jayne M, Ma Y, Wong C (2006) Cocaine cues and dopamine in dorsal striatum: mechanism of craving in cocaine addiction. J Neurosci 26:6583-6588.

Whishaw IQ, Gorny B, Foroud A, Kleim JA (2003) Long-Evans and Sprague-Dawley rats have similar skilled reaching success and limb representations in motor cortex but different movements: some cautionary insights into the selection of rat strains for neurobiological motor research. Behav Brain Res 145:221-232.

Yaka R, Thornton C, Vagts AJ, Phamluong K, Bonci A, Ron D (2002) NMDA receptor function is regulated by the inhibitory scaffolding protein, RACK1. Proc Natl Acad Sci USA 99:5710-5715.

Yaka R, He DY, Phamluong K, Ron D (2003a) Pituitary adenylate cyclaseactivating polypeptide (PACAP(1-38)) enhances $N$-methyl-D-aspartate receptor function and brain-derived neurotrophic factor expression via RACK1. J Biol Chem 278:9630-9638.

Yaka R, Phamluong K, Ron D (2003b) Scaffolding of Fyn kinase to the NMDA receptor determines brain region sensitivity to ethanol. J Neurosci 23:3623-3632.

Yaka R, Tang KC, Camarini R, Janak PH, Ron D (2003c) Fyn kinase and NR2B-containing NMDA receptors regulate acute ethanol sensitivity but not ethanol intake or conditioned reward. Alcohol Clin Exp Res 27:1736-1742.

Yin HH, Knowlton BJ (2006) The role of the basal ganglia in habit formation. Nat Rev Neurosci 7:464-476.

Yin HH, Lovinger DM (2006) Frequency-specific and D2 receptormediated inhibition of glutamate release by retrograde endocannabinoid signaling. Proc Natl Acad Sci USA 103:8251-8256.

Zucker RS, Regehr WG (2002) Short-term synaptic plasticity. Annu Rev Physiol 64:355-405. 